\title{
Diacronie
}

Studi di Storia Contemporanea

$N^{\circ} 17,1 \mid 2014$

Periferie. Cultura, economia, politica

\section{Fragmentación política del centrismo Español,}

\section{1-1982}

De la UCD al CDS en el caso de Zamora

\section{Darío Diez Miguel}

\section{OpenEdition}

\section{Journals}

Edición electrónica

URL: http://journals.openedition.org/diacronie/1082

DOI: 10.4000/diacronie.1082

ISSN: 2038-0925

\section{Editor}

Association culturelle Diacronie

Referencia electrónica

Darío Diez Miguel, « Fragmentación política del centrismo Español, 1981-1982 », Diacronie [En línea], N - 17, 1 | 2014, documento 9, Puesto en línea el 01 marzo 2014, consultado el 20 abril 2019. URL :

http://journals.openedition.org/diacronie/1082 ; DOI : 10.4000/diacronie.1082 


\section{Diacronie}

9/

\section{Fragmentación política del centrismo Español, 1981-1982 \\ De la UCD al CDS en el caso de Zamora}

Darío DIEZ MIGUEL*

El presente artículo se centra en el estudio de la descomposición de Unión de Centro Democrático (UCD) y su interrelación con la génesis del Centro Democrático y Social (CDS) en la provincia de Zamora, tomando como referencia la dimensión nacional de ambos procesos dentro del contexto de la Transición española. Para ello, nos serviremos de fuentes documentales directas y artículos de prensa. Con el fin de entretejer nuestra argumentación nos moveremos en el marco teórico desarrollado por Jonathan R. Hopkin en su estudio clásico sobre la crisis de UCD. Valoraremos el peso de las luchas de poder y las convicciones ideológicas como causas de la descomposición de UCD y formación posterior del CDS.

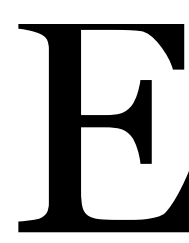

n nuestro estudio, recorreremos los últimos compases de la Transición española, centrándonos en la trayectoria del partido político en el gobierno, la UCD, creada en el entorno del presidente Adolfo Suárez hacia 1977.

La UCD fue un conglomerado heterogéneo de familias ideológicas y políticas que aglutinaba a democristianos, socialdemócratas, liberales y con una fuerza singular, a los sectores reformistas del régimen franquista. A pesar de los éxitos electorales de 1977 y 1979, no se consiguió lograr la consolidación de este partido político, que desapareció en 1983 tras un largo período de tensiones internas, recrudecidas al hilo de la dimisión de Suárez a comienzos de 1981 y su abandono definitivo de UCD apenas un año después para fundar el CDS. A través de la exposición 
de este estudio de caso, esperamos contribuir a la comprensión de este complejo proceso, la crisis de UCD, que afectó sensiblemente a la consolidación del sistema de partidos español.

A día de hoy, el debate historiográfico acerca de la entidad de UCD como partido político permanece abierto. Si para algunos la UCD fue una herramienta política que únicamente podía servir para llevar a cabo la Transición, para otros la consolidación hubiera sido posible si el comportamiento político de sus miembros hubiese sido distinto $^{1}$. Sin embargo, a pesar de la cantidad de estudios referentes a UCD, no es exagerado afirmar que su análisis ha recibido un tratamiento historiográfico desigual. Aunque el papel político de UCD ha sido espléndidamente sintetizado por numerosos autores $^{2}$, persiste cierto vacío, en el estudio de la intrahistoria del partido en los niveles provinciales y locales 3 . Asimismo, los partidos surgidos de la descomposición centrista, como el CDS, el PDP o el PAD, apenas han suscitado el interés de los investigadores, biógrafos o periodistas 4 . La explicación más plausible a estas ausencias, no sólo tiene

\footnotetext{
* Este artículo se enmarca dentro del proyecto de tesis doctoral titulado "Adolfo Suárez y el Centro Democrático y Social: 1982-1991", tutelada por el profesor titular de la Univ. de Valladolid José-Vidal Pelaz López. La base de este trabajo son las fuentes primarias de UCD existentes en el Archivo Histórico-Provincial de Zamora (AHPZ), fondo UCD, de aproximadamente 80 cajas y varios vademécum. Dicho fondo ha sido clasificado ex profeso en el marco de esta investigación, si bien, está a expensas de ser inventariado definitivamente por parte del AHPZ. A lo largo de este trabajo, se han omitido los nombres propios de algunos miembros de la UCD de Zamora, cuando se ha considerado que podía ser doloso u ofensivo para los mismos y no afectaba a nuestra argumentación. Con anterioridad, este fondo ha sido trabajado por GONZÁLEZ CLAVERO, Mariano, Fuerzas Políticas en el Proceso Autonómico de Castilla y León 1977-1983, Tesis Doctoral, Univ. de Valladolid, 2002.

${ }^{1}$ Una interesante síntesis de esta cuestión es: MOLINA JIMÉNEZ, Daniel, «La desintegración de la UCD: estado de la cuestión», in El Futuro del Pasado, 2, 2011, pp. 255-264, URL: $<$ http://dialnet.unirioja.es/servlet/articulo?codigo $=3697369>$ [consultado el 10 de junio de 2006]. Para una perspectiva política general, se aconseja la lectura de: POWELL, Charles, España en democracia, Barcelona, Plaza \& Janes, 2001.

2 ALONSO CASTRILLO, Silvia, Apuesta del centro: una historia de UCD, Madrid, Alianza Editorial, 1996; HOPKIN, Jonathan Richard, El partido de la transición. Ascenso y caída de la Unión de Centro Democrático, Madrid, Acento, 2000; HUNEEUS, Carlos, La Unión de Centro Democrático y la Transición a la Democracia en España, Madrid, CIS, 1985, etc. Desde el punto de vista de las memorias políticas, quizá la obra más interesante resulta, CALVOSOTELO, Leopoldo, Memoria Viva de la Transición, Barcelona, Plaza \& Janés, 1990.

3 Destacan las obras de periodistas y comentaristas políticos de la época como JÁUREGUI, Fernando, SORIANO, Manuel, La otra historia de UCD, Madrid, Emiliano Escolar, 1980; FIGUERO, Javier, UCD. La empresa que creó Adolfo Suárez, Madrid, Grijalbo, 1981; CHAMORRO, Eduardo, Viaje al Centro de UCD, Barcelona, Planeta, 1981.

${ }_{4}^{4} \mathrm{El}$ tratamiento historiográfico del CDS ha sido prácticamente nulo hasta el momento, aparte del breve recorrido que realizan sobre él los biógrafos de A. Suárez destacando la obra del profesor FUENTE ARAGONÉS, Juan Francisco, Adolfo Suárez: la historia que no se contó, Planeta, 2011. Asimismo, cabe destacar la visión de conjunto de, QUIROSA-CHEYROUZE MUÑOZ, Rafael, «El Centro Democrático y Social. Auge y caída de un proyecto político (19821996)» in MATEOS LÓPEZ, Abdón, SOTO CARMONA, Álvaro (dir.es), Historia de la época socialista: 1982-1996, Madrid, Ed. Sílex, 2013, pp. 405-430. Sobre sus primera etapa, FERNÁNDEZ AMADOR, Mónica, QUIROSA-CHEYROUZE MUÑOZ, Rafael, «La creación de
} 
que ver con lo reciente de estos fenómenos, también nos habla de que las fuentes primarias para el análisis de esta realidad no son muy abundantes y las existentes no han sido objeto de un estudio sistemático hasta el momento.

De este modo, a la hora de analizar las causas de la fractura de la UCD en Zamora y su relación con la constitución del CDS en la provincia, durante el verano de 1982, nos hemos encontrado con la inexistencia de trabajos específicos ${ }^{5}$. En el transcurso de nuestra investigación, por un lado, ha resultado revelador el análisis de las trayectorias de quienes encabezaron la gestación a nivel local-provincial del nuevo partido de Adolfo Suárez, -el CDS-, a lo largo de sus dos últimos años de pertenencia a UCD. Por otra parte, el marco teórico desarrollado por Jonathan R. Hopkin en su análisis de la descomposición de UCD nos ha proporcionado sugerentes claves interpretativas para abordar los distintos sucesos que tuvieron lugar6.

\section{Génesis de la crisis en la UCD zamorana}

La UCD había resultado vencedora indiscutible en los procesos electorales de 1977 y 1979 celebrados en Zamora, con unos porcentajes7 que superaban holgadamente la media nacional, erigiéndose en el grupo político más poderoso de la provincia. En 1977, UCD obtuvo en Zamora un 46,59\%, frente a un 34,44\% a nivel nacional; en 1979, alcanzó el 50, 57\% frente a un 34,84\%. Incluso, en las elecciones de 1982, Zamora fue una de las pocas provincias donde UCD obtuvo representación, gracias a la elección

Centro Democrático y Social en 1982» in QUIROSA-CHEYROUZE Y MUÑOZ, Rafael, (ed.) Los partidos en la Transición, Madrid, Biblioteca Nueva, 2013, pp. 201-220.

5 Hay numerosos estudios de la etapa final de la UCD a nivel provincial y regional como los análisis de Carlos Navajas Zubeldia para el caso riojano, NAVAJAS ZUBELDIA, "Desconcierto" y "zozobra". La segunda transición autonómica en la Rioja (1982-1983), in Actas del III Simposio de Historia Actual. Logroño, 26-28 de octubre de 20oo, Logroño, Gobierno de La Rioja, Instituto de Estudios Riojanos, 2002, pp. 285-320; o para el caso de Valencia, aunque no se centre especialmente en la crisis política del partido a nivel interno, GASCÓ ESCUDERO, Patricia, UCD-Valencia. Estrategias y grupos de poder político, Valencia, Universidad de Valencia, 2011, pp. 69-91.

6 En este marco teórico, Jonathan Hopkin pone en relación el desarrollo de fórmulas alternativas de integración política que den respuesta al descontento interno, ejercicios de "voz", con la existencia de "opciones de salida", que se negocian y ejecutan cuando fracasa la primera vía, «la incapacidad para aumentar su influencia en las decisiones [...] incrementó indudablemente el atractivo de la estrategia de "salida"». Hopkin utilizó este modelo, especialmente, para explicar las "deserciones" de los sectores conservadores del partido centrista y subrayar que «los desacuerdos sobre objetivos ideológicos no constituyen una explicación suficiente para la gravedad del conflicto interno del partido», HOPKIN, Jonathan Richard, El partido de la transición. Ascenso y caída de la Unión de Centro Democrático, cit., p. 292 et seq.

7 Base de datos electorales del Ministerio del Interior de España; in URL:

$<$ http://www.infoelectoral.mir.es > [consultado el 1 de noviembre de 2013]. 
como diputado de Luis Ortiz González. Los diputados y senadores electos por Zamora en 1977 y 1979 fueron:

\begin{tabular}{|l|l|l|l|}
\hline \multicolumn{2}{|l|}{ DIPUTADOS 1977} & \multicolumn{2}{l|}{ SENADORES 1977 } \\
\hline UCD & JOSE A.OTERO MADRIGAL & UCD & VALERIANO ENRÍQUEZ GONZÁLEZ \\
\hline UCD & MODESTO ALONSO PELAYO & UCD & VICTOR M. CARRASCAL FELGUEROSO \\
\hline AP & FEDERICO SILVA MUÑOZ & UCD & LUIS RODRÍGUEZ SAN LÉON * \\
\hline PSOE & DEMETRIO MADRID LOPEZ & PSOE & MANUEL ALONSO NOVO \\
\hline
\end{tabular}

\begin{tabular}{|l|l|l|l|}
\hline \multicolumn{2}{|l|}{ DIPUTADOS 1979} & \multicolumn{2}{l|}{ SENADORES 1979} \\
\hline UCD & VICTOR M. CARRASCAL FELGUEROSO & UCD & LUIS RODRÍGUEZ SAN LÉON * \\
\hline UCD & CESAR MARTIN MONTES * & UCD & RICARDO RODRÍGUEZ CASTANÓN \\
\hline UCD & JESUS PEREZ LOPEZ *8 & UCD & ONÉSIMO LÓPEZ CHILLÓN \\
\hline PSOE & DEMETRIO MADRID LOPEZ & AP & CARLOS PINILLA TURIÑO \\
\hline
\end{tabular}

Fue precisamente en este contexto de éxito político, el momento en el que se gestó el origen de la futura división y descomposición del partido a nivel provincial. El grupo de UCD Zamora evidenciaba síntomas de desunión desde 1979, apenas año y medio después de la formalización definitiva de sus órganos de gobierno, cuando el diputado Víctor Carrascal Felgueroso fue elegido presidente en la II Asamblea Provincial9 del partido, con la ausencia de más de la mitad de los convocados y la negativa del antiguo presidente provincial, Luis Rodríguez San León ${ }^{10}$ a ser incluido en las listas. Luis Rodríguez San León había presentado su dimisión el 3 de mayo de 1979¹1, poco después de ser elegido senador por UCD en la circunscripción zamorana. Sobre, la nueva dirección política del partido en Zamora comentaba un destacado dirigente centrista:

\footnotetext{
8 Con un asterisco señalamos aquellos parlamentarios de UCD que se integraron en el CDS en agosto de 1982. Es necesario hacer una breve semblanza política y profesional de los que van a ser los protagonistas de este suceso. Por un lado, se encontraban el senador Luis Rodríguez San León quien era un conocido empresario zamorano y director de diversas asociaciones locales, César Martín Montes, palentino, vinculado al Servicio Nacional de Inspección de las Administraciones Locales, trabajando en la provincia de Zamora desde 1966, y Jesús Pérez López, zamorano, asesor jurídico de la UCD provincial desde sus inicios. Por otra parte, Víctor Carrascal Felgueroso, político oriundo de Madrid, con amplia formación académica y vinculado a Ignacio Camuñas en el tardofranquismo, José Andrés Díez de Ulzurrun Mosquera ( ${ }^{0} 4$ en la listas al congreso de 1979), zamorano, ingeniero técnico agrario en el I.R.Y.D.A. desde 1966, y Onésimo López Chillón, zamorano, agricultor y promotor de la Asociación Independiente de Ganaderos y Labradores de Zamora. AHPZ, Fondo UCD, caja 21, Dossier de la Secretaría de Información de UCD, Biografías de Diputados y Senadores.

9 El País, 17 julio 1979.

${ }^{10}$ Luis Rodríguez San León fue elegido presidente provincial de UCD en Zamora el 9 diciembre 1977. AHPZ, Fondo UCD, caja 21, Dossier de la Secretaría de Información de UCD.

${ }^{11}$ AHPZ, Fondo UCD, caja 2, Carta de Dimisión de Luis Rodríguez San León.
} 
A. No, no existían [las baronías], curiosamente vinieron a continuación. Es decir, de los que estaban que subieron a la ejecutiva en la asamblea siguiente [1979] [...] es ahí donde comenzó el primer error y la primera caída de la UCD provincial [...] la historia de este país es que todo el mundo quiere ser diputado, pero por ej. los que crearon la UCD, todo el mundo quería ser diputado por Madrid, pero claro, el presidente Suárez decía, bueno, es que en la lista de Madrid son 25, yo lo que no puedo hacer es meter a 48 [...] todo lo que ha ocurrido en UCD es una razón completamente personal [...]

Q. Entonces en estas luchas no fue muy importante asuntos ideológicos ni de organización. Simplemente la lucha por el poder.

A. La lucha por el poder...12

La situación de la UCD en Zamora, hacia 1979, estaba atravesada por una fractura política entre el cuadro directivo saliente (encabezado por Luis Rodríguez San León) y el entrante, (con Víctor Carrascal Felgueroso al frente), que asimismo dividió a las bases y cargos electos del partido durante los años siguientes. La lucha por el control del aparato provincial y los problemas en la composición de las listas electorales fueron las causas subyacentes de este distanciamiento.

En 1981, las diferentes tensiones existentes dentro de la UCD, más o menos soterradas hasta entonces, estallaron a raíz de la dimisión del presidente y líder centrista, Adolfo Suárez, a finales de enero; una dimisión, en la que se entremezclaban, por un lado, la contestación interna en el seno de su propio partido y por otra parte, la fuerte oposición del PSOE, el distanciamiento de la Corona, la presión de los "poderes fácticos”, etc. El ambiente de división en UCD tuvo su reflejo en el II Congreso Nacional, (celebrado entre el 6 y el 8 de febrero de 1981), en el que mostraron su fuerza dos grandes corrientes ${ }^{13}$ : la oficialista/suarista/aparato de UCD, - que consiguió la presidencia del partido para Agustín Rodríguez Sahagún y la secretaría general para Rafael Calvo Ortega ${ }^{14-}$, y los llamados "críticos" (de tendencia más conservadora). Aunque primó la posición suarista, era imposible obviar el fuerte ejercicio de

\footnotetext{
${ }^{12}$ Archivo Gunther. Interview undertaken by Richard Gunther in 1983 (june). C 22, pp. 4-5.

${ }^{13}$ A este respecto hay cierta discusión entre los autores que han investigado, desde distintas perspectivas, la historia de UCD. Para Carlos Huneeus, desde la prensa se exageró el poder del aparato de UCD atribuyendo a Suárez un control sistemático del partido desde fuera, HUNEEUS, Carlos, La Unión de Centro Democrático y la Transición a la Democracia en España, cit., p. 337. En abierta oposición a esta interpretación, ALONSO CASTRILLO, Silvia, Apuesta del centro: una historia de UCD, cit., p. 506 et seq.

${ }_{14}$ Ambos, elegidos en aquel II Congreso, habían sido ministros en los gobiernos de Suárez y en 1982, pasaron a formar parte del Comité Nacional del CDS.
} 
contestación interna. Asimismo, en aquellas fechas, se confirmó a Leopoldo CalvoSotelo como relevo del dimisionario Adolfo Suárez en la presidencia de gobierno ${ }^{15}$.

En el ámbito provincial, a comienzos de julio de 1981, tuvo lugar un proceso de renovación de cargos que repitió en sus formas y enfrentamientos lo sucedido a nivel nacional, si bien, con algunos matices particulares. La preparación de la III Asamblea Provincial de Zamora, celebrada en julio de 1981, ahondó la división existente en la provincia. Los dos bloques que se habían fraguado en 1979 dentro del partido estaban a la altura de 1981 perfectamente perfilados y presentaron sus listas respectivas en abierta oposición ${ }^{16}$. Por un lado, se presentaba una lista apoyada por quienes habían llevado las riendas de la UCD en Zamora desde 1979, entre ellos el presidente provincial Víctor Carrascal Felgueroso ${ }^{17}$. Al frente de esta lista se encontraba el Ministro de Obras Públicas, Luis Ortiz González, uno de los hombres de gobierno más cercanos a Leopoldo Calvo-Sotelo. Frente a ellos, se había constituido una auténtica plataforma opositora auspiciada por aquellos que no participaron en la II Asamblea Provincial18. Estaban encabezados por el senador Luis Rodríguez San León, los diputados César Martín Montes, Jesús Pérez López y el Presidente de la Diputación Provincial, José Miguel López Martínez, quienes entre junio y julio de 1981 firmaron una serie de artículos en El Correo de Zamora en los que presentaban sus propuestas de renovación de los órganos de gobierno provinciales ${ }^{19}$ :

La lista ahora llamada "oficialista" (observen la paradoja), para la que se obtuvieron refuerzos foráneos mediante el aterrizaje de conocidos políticos

\footnotetext{
${ }^{15}$ Anteriormente, Leopoldo Calvo-Sotelo había ocupado diversos ministerios en los gobiernos de Carlos Arias Navarro y Adolfo Suárez. Asimismo, había sido el encargado de gestionar la formación de la coalición que dio lugar a la UCD, formación en la que no se adscribía a ninguna familia o facción.

16 «UCD renueva este fin de semana 12 ejecutivas provinciales [...] también en Zamora se presenta una situación de enfrentamiento», en El País, 4 julio 1981.

17 «Soy una de las personas que más ha trabajado en la aproximación [...] desde el principio en el que se planteó la presidencia de Luis Ortiz yo dije que iba a estar a su lado y en este apoyo me mantengo asumiendo los errores que haya podido cometer»; cfr., AHPZ, Fondo UCD, caja 64, Acta del Comité Ejecutivo Provincial de UCD Zamora, 6 marzo 1982, p. 3.

${ }_{18}$ Un fenómeno de división interna equiparable al de Zamora, por su premura y consistencia, es el caso de Segovia en el que una de las causas de la ruptura es la integración o no de Segovia en la naciente comunidad autónoma castellano-leonesa, PÉREZ LÓPEZ, Pablo y GONZÁLEZ CLAVERO, Mariano, La transición en Segovia, Palencia, Cálamo, 2007, pp. 146-153, p. 189 et seq.

19 Dichos artículos se encontraban como documentos anejos a una de las fichas de expulsión del Comité Disciplinario de UCD, en agosto de 1982: "Desde el Congreso de Palma», in El Correo de Zamora, 30 junio 1981, "Contestando al ministro del M. O. P. U.», in El Correo de Zamora, 3 julio 1981 y «Ante la Asamblea de UCD», in El Correo de Zamora, 4 julio 1981. AHPZ, Fondo $U C D$, caja 65, Dossier de Expulsados.
} 
madrileños [...] De cara a la asamblea del 5 de julio las posiciones están tomadas.

Continuismo o renovación. Colonialismo o autonomía política provincial ${ }^{20}$.

Sobre Luis Ortiz añadían, «no nos dice qué motivaciones de conciencia le indujeron a acordarse de Zamora, precisamente de Zamora, para el ejercicio de su misión salvadora ${ }^{21}$. A diferencia de lo que ocurría en el resto de España, en Zamora, el enfrentamiento político en el seno de UCD se identificaba sólo parcialmente con "el oficialismo" y con "el sector crítico". Como vemos, las luchas de poder a nivel local y provincial entre los líderes de la UCD cobraron forma en base a la polémica entre los que se consideraban defensores de los intereses de Zamora frente a los políticos colocados desde Madrid -cuneros-, y sólo a posteriori, desembocaron en la vinculación de los dos bloques a unas u otras de las familias del partido, en función de su afinidad ideológica. El grupo encabezado por Luis Rodríguez San León se posicionaba políticamente en abierta discrepancia con el llamado sector crítico y apostaba por el resurgir, desde la UCD, de una oferta política de centro-centro ${ }^{22}$, en sintonía con los hombres más cercanos de Adolfo Suárez. Sin embargo, su poder no residía tanto en la presión del aparato suarista del partido, como en la importancia de los cargos que desempeñaban y su capacidad de influencia a nivel local y provincial.

\section{De los últimos intentos de integración a la división definitiva}

Los resultados de la II Asamblea Provincial de Zamora depararon una apretada victoria, - 247 votos frente a $217^{23}$-, de la lista encabezada por Luis Ortiz González, quien se convirtió en el nuevo presidente de la UCD zamorana, junto a él, José Díez de Ulzurrun Mosquera, resultó elegido secretario general, apoyados por el ex presidente provincial Víctor Carrascal Felgueroso. Asimismo, como miembros con voz pero sin voto de la nueva ejecutiva -en tanto que parlamentarios de la provincia - se encontraban Luis Rodríguez San León, Jesús Pérez López y César Martín Montes²4. A partir de entonces, la situación en la UCD de Zamora, se fundió totalmente con la del

20 In El Correo de Zamora, 30 junio 1981; cfr., AHPZ, Fondo UCD, caja 65, Dossier de Expulsados.

${ }^{21}$ In El Correo de Zamora, o3 julio 1981; cfr., AHPZ, Fondo UCD, caja 65, Dossier de Expulsados.

22 In El Correo de Zamora, 30 junio 1982; cfr., AHPZ, Fondo UCD, caja 65, Dossier de Expulsados. Una amplia polémica sobre la definición de centro-centro, centro-izquierda y las acusaciones de pseudoprogresismo, en RODRÍGUEZ SAHAGÚN, Agustín, «De la división a la desnaturalización» en Historia de la Transición, Grupo 16, 1984, pp. 708 et seq.

${ }^{23}$ In El País, o7 julio 1981.

24 AHPZ, Fondo UCD, caja 21, Dossier de la Secretaría de Información de UCD. 
centrismo nacional y el pulso político del grupo centrista provincial pasó a ser un reflejo sintomático de la convulsa situación de la UCD entre los años 1981 y $1982^{25}$.

En el otoño de 1981, tuvo lugar la dimisión del sector socialdemócrata del partido centrista, tanto a nivel nacional, como provincial. Ricardo Rodríguez Castañón ${ }^{26}$, senador socialdemócrata por Zamora, comunicaba oficialmente su renuncia a la militancia centrista indicando explícitamente que la situación del partido en Zamora, «no puede sustraerse a la realidad del Partido a nivel Nacional»27. La marcha de la facción socialdemócrata se solapaba con los cambios en la presidencia/secretaría general de UCD de noviembre del $1981^{28}$, tras el fracaso centrista en las elecciones autonómicas gallegas. Leopoldo Calvo-Sotelo se hacía cargo del partido, en lugar de Agustín Rodríguez Sahagún, e Íñigo Cavero recalaba en la secretaría general sustituyendo a Rafael Calvo Ortega. De este modo, el sector suarista era relegado del poder.

La reunión del Comité Ejecutivo Provincial celebrada con posterioridad a estas dimisiones y encabezada por el ministro Luis Ortiz González, consciente de la peligrosa situación que se estaba generando, trató de quitar hierro a los problemas mostrando su total afinidad a la nueva dirección del partido: «es necesario transmitir la situación de noramalidad [sic] a todos los sectores de nuestra provincia» ${ }^{29}$. A aquella reunión del Comité Ejecutivo no asistieron ni Jesús Pérez López, ni César Martín Montesº; por aquellas fechas, miembros del sector suarista comenzaron a ausentarse también de las reuniones de la ejecutiva nacional ${ }^{31}$. Sin embargo, todavía había una posibilidad de convergencia de intereses entre las dos facciones.

Por un lado, de aquellas reuniones de la ejecutiva provincial se trasluce que la mayor preocupación era evitar que se consumase la división del partido existente en la

${ }^{25}$ El paradigma de esta nueva fase de la crisis de UCD es la sustitución del recurso a la "voz", por la estrategia de la "salida"; cfr., HOPKIN, Jonathan Richard, El partido de la transición. Ascenso y caída de la Unión de Centro Democrático, op. cit. p. 294. Lógicamente, no sucedió así en todas las provincias, por ejemplo en la vecina León, «en 1982 el leonés [Martin Villa] seguía siendo presidente del Consejo Político Provincial de UCD [...] Las disensiones de Madrid, llegan tarde a León»; cfr., FERNÁNDEZ, Pedro Víctor, La transición en León, Salamanca, Diputación de León, 2008.

${ }_{26}$ Sobre su participación en el PAD de Francisco Fernández Ordóñez, jefe del ala socialdemócrata de UCD y ministro en diversos gobiernos de A. Suárez y L. Calvo-Sotelo, véase: Partido de Acción Democrática. Congreso Constituyente, Madrid, PAD, 1982, pp. 17-42.

27 AHPZ, Fondo UCD, caja 65, Dossier de Expulsados. Carta de Rodríguez Castañón, 13-11-1981.

${ }^{28}$ Véase: HUNEEUS, Carlos, La Unión de Centro Democrático y la Transición a la Democracia en España, op. cit., pp. 373 et seq.

29 Ibidem, p. 3.

30 Esta reunión constituye la última de la que tenemos constancia de la presencia de Luis Rodríguez San León. AHPZ, Fondo UCD, caja 64, Acta de la reunión del Comité Ejecutivo Provincial de U.C.D. de Zamora, 11 diciembre 1981, 22 enero 1982 y 6 marzo 1982.

${ }^{31}$ Supone el abandono de Suárez de facto del Comité Ejecutivo de UCD; cfr., ABELLA, Carlos, Adolfo Suárez: El hombre clave de la Transición, Madrid, Espasa, 2006, p. 543. 
provincia, con el fin de no perjudicar aún más al partido/gobierno estatal ${ }^{32}$, - hemos de recordar que el presidente provincial Luis Ortiz González era en ese momento ministro de Obras Públicas del gobierno de Leopoldo Calvo-Sotelo, a su vez, presidente del partido centrista. Por otra parte, en la dialéctica entre aumentar su presencia en los órganos directivos del partido o abandonarlo definitivamente, al hilo de los desarrollos teóricos de J. Hopkin aplicados a UCD - el grupo del senador Luis Rodríguez San León estaba en condiciones - debido a la importancia de sus cargos y su liderazgo a nivel provincial/local, de exigir nuevas fórmulas de participación:

Tras largas y extensas conversaciones con los Parlamentarios Luis Rodríguez San León, César Martín Montes y Jesús Pérez López, y con el Presidente de la Diputación, para tratar de llegar a un acuerdo en el que se lograra la plena integración de estos en el seno del Partido, se ha elaborado un documento que se somete a consideración de este Comité Ejecutivo para que adopte los acuerdos correspondientes 33 .

En este documento, se proponía la creación de un Comité de Coordinación y Acción Provincial que contase con la presencia de todos los grandes cargos provinciales y tuviese destacados poderes en la formación de las listas (una de las cláusulas requería la inclusión de los parlamentarios electos en 1979 en las futuras listas de forma automática). Aunque se realizó una votación que resultó favorable a la formación del nuevo Comité - salvo el apartado referente a la inclusión, por defecto, de los parlamentarios elegidos en 1979 en las nuevas listas - esta fórmula de integración, consensuada en cierto modo por ambas facciones, llegó demasiado tarde; suponía el último intento, al margen ya de las fórmulas estatutarias, de integración política. Una anotación a mano, junto al acta de aquella reunión resumía el estado de ánimo dentro del partido: «Desde UCD, no podemos permitirnos el lujo de pregonar la democracia, el progreso, la paz. Yo pido a todos, generosidad, y que nadie se mire a sí mismo, sino a los demás. Ni vencedores, ni vencidos. No todos los problemas están resueltos» 34 .

32 AHPZ, Fondo UCD, caja 64, Acta de la reunión del Comité Ejecutivo Provincial de U.C.D. de Zamora, 11 diciembre 1981, 22 enero 1982 y 6 marzo 1982.

33 AHPZ, Fondo UCD, caja 64, Acta de la reunión del Comité Ejecutivo Provincial de U.C.D. de Zamora, 6 marzo 1982.

34 AHPZ, Fondo UCD, caja 5, Acta de la reunión del Comité Ejecutivo Provincial de U.C.D. de Zamora del 06 marzo 1982 con anotaciones manuscritas. De cara a la opinión pública se daba una versión maquillada de los hechos: «Ayer día 5 de julio se cumplió el primer aniversario de la elección de Luis Ortiz González como presidente de UCD en Zamora, [...] La elección fue dura y reñida y provocó un cierto malestar en algunos de los miembros de la candidatura derrotada, origen, quizás, de las discrepancias que, a lo largo del año, se han manifestado, sobre todo por parte de los parlamentarios que quedaron fuera del Comité Ejecutivo [...] El señor Ortiz 
En las semanas siguientes, el bloque de parlamentarios enfrentado a la cúpula provincial35 acentuó sus actividades como un grupo autónomo, cada vez más abiertamente explícito en sus acusaciones al ejecutivo centrista, identificado con la dirección provincial del partido36:

[...] ha ignorado, marginado, y menospreciado al Presidente del Partido, y a los Parlamentarios, cargos Provinciales y Locales, miembros del Comité Ejecutivo y militantes en general que no forman parte del grupo perdedor en la Asamblea Provincial de 1981, que encabeza el Senador D. Luis Rodríguez San León quien recientemente abandonó UCD para incorporarse al nuevo Partido Centro Democrático y Social [...] Tal connivencia, que ya quedó apuntada en el apartado anterior, se ha materializado en numerosas visitas realizadas por ambos a pueblos de la Provincia; visitas [...] a las que nunca fueron invitados el Presidente o Secretario Provincial del Partido, Parlamentarios del otro grupo etc. Estas visitas han quedado reflejadas sobradamente, en la radio y prensa local [...]37.

De este modo, los posicionamientos de ambos grupos, a la altura de la primavera de 1982 estaban perfectamente clarificados. El Comité Ejecutivo Provincial se identificaba con la política gubernamental y la gestión de UCD por parte de Leopoldo Calvo-Sotelo; por su parte, el grupo encabezado por Luis Rodríguez San León, partidario del centrocentro, agotó definitivamente la búsqueda de una alternativa integradora en el seno de la UCD provincial, expresión de "voz", al encontrar un nuevo cauce de representación política junto a otros cuadros provinciales de UCD, a nivel nacional, a lo largo de junio y julio de 1982, cuya demanda principal era la vuelta de Adolfo Suárez a la presidencia

González considera que sus tres aspiraciones se han visto cumplidas [...]», in El Correo de Zamora, 6 julio 1982. AGCYL, Gabinete de información, caja 243, Informe Diario $\mathrm{n}^{\mathrm{o}} 317$.

35 Entre la documentación adjunta a uno de los expedientes de expulsión, se hallaban los siguientes artículos, destinados a demostrar las actividades de este grupo: «Los parlamentarios Pérez López, Martín Montes y Rodríguez San León, con el ministro de Trabajo y S. S. Les acompañaban el presidente de la Diputación y el alcalde de Toro» en El Correo de Zamora, 27 marzo 1982; "Acompañado por el senador Luis Rodríguez San León. El Ayuntamiento de Cubillos, visita al presidente de la Diputación Provincial» in El Correo de Zamora, 28 marzo 1982; «El presidente de la Diputación y el senador Rodríguez San León, con el subsecretario del ministerio de agricultura» in El Correo de Zamora, 19 junio 1982. AHPZ, Fondo UCD, caja 65, Dossier de Expulsados.

${ }^{36}$ Pasando de calificar como "eficiente" a Leopoldo Calvo-Sotelo en «Desde el Congreso de Palma», in El Correo de Zamora, 30 junio 1981 in AHPZ, Fondo UCD, caja 65, Dossier de Expulsados; a pedir su dimisión en mayo de 1982, «Luis Rodríguez San León apuntaba que queríamos haberle dicho que se marchara y lo dejara. En un país medianamente serio, el presidente no espera a informar, sino que se marcha a su casa», in $A B C, 28$ mayo 1982.

37 Cargos para la incoación de un expediente disciplinario. AHPZ, Fondo UCD, caja 65, Dossier de Expulsados, Informe 6 agosto 1982. 
del partido ${ }^{38}$. Leopoldo Calvo-Sotelo, tras el fracaso de las elecciones andaluzas de mayo del 82', había cedido la presidencia de UCD a Landelino Lavilla. La situación del partido de gobierno, a esas alturas, era desesperada, teniendo lugar incluso unas últimas negociaciones entre Suárez, Calvo-Sotelo y Lavilla que no dieron ningún resultado.

La "opción de salida” primó sobre cualquier otra solución cuando el líder centrista, Adolfo Suárez, abandonó UCD y creó el CDS, institucionalizando esta mezcla de suarismo y malestar político existente en el verano de 198239. Así, la creación de este nuevo partido permitió dar una solución razonable a las numerosas luchas de poder enquistadas en el conglomerado centrista, cuyas causas, como estamos viendo se remontaban años atrás:

\begin{abstract}
ANTE LA CRISIS DE UCD [doble subrayado] [...] CONSCIENTES [subrayado] de que las causas originantes de estos hechos son de índole muy diversa, y vienen de tiempo atrás sin que puedan reconducirse a una sola por la heterogeneidad de las mismas $4^{40}$.
\end{abstract}

Cuando se impuso "la opción de salida" sobre la negociación, las relaciones en el seno de UCD-Zamora estaban muy deterioradas y es difícil achacarlas a cuestiones meramente ideológicas. Las bajas de Luis Rodríguez San León ${ }^{41}$, César Martín Montes, Jesús Pérez López y José Miguel López Martínez estuvieron acompañadas de dimisiones y expulsiones de UCD a lo largo de toda la provincia42, habiéndose identificado más de una decena de incoaciones de expedientes por parte del Comité de Disciplina Provincial, resueltas definitivamente en una reunión mantenida el 9 de

${ }^{38}$ Serafín Abilio Martínez Fernández, Secretario Regional de UCD de Asturias, remitía a la secretaría provincial de Zamora un texto elaborado en Madrid, en una reunión mantenida días atrás, por unos 30 secretarios provinciales en el que se concluía: «es necesario adecuar la dirección del Partido al momento presente, estableciendo un claro liderazgo en el mismo, que, consideramos, debe recaer en la persona del Fundador del Partido, Adolfo Suárez González». AHPZ, Fondo UCD, caja 5. En la misma línea, aunque sin aludir explícitamente a Adolfo Suárez, véase: Archivo Histórico Provincial de Soria (AHPS), Fondo Unión de Centro Democrático, caja 3350, Conclusiones del Comité Ejecutivo Regional de UCD-Asturias del 8 junio 1982.

$39 \mathrm{El}$ depósito de los estatutos del partido tuvo lugar a finales de julio de 1982, quedando inscrito definitivamente el 23 de agosto del mismo año. Ministerio del Interior, Registro de Partidos Políticos, Dossier CDS, año 1982, protocolo notarial n ${ }^{0} 1771$.

$4^{\circ}$ AHPS, Fondo UCD, caja 3350, Conclusiones del Comité Ejecutivo Regional de UCD-Asturias, 8 junio 1982, p. 2.

${ }^{41}$ Cabe añadir, que Luis Rodríguez San León fue uno de los primeros políticos ucedistas que solicitó su baja en UCD para integrarse en el CDS: «y el primer ucedista que solicitó la dimisión de Leopoldo Calvo Sotelo tras el fracaso de la UCD en las elecciones andaluzas», in El Periódico de Catalunya, 30 julio 1982.

42 Asimismo hubo muestras de inquebrantable adhesión a la UCD provincial por parte de alcaldes, concejales y militantes centristas, AHPZ, Fondo UCD, caja 5. 
agosto de 198243. Las acusaciones versaban sobre todo acerca de la pertenencia de los militantes expulsados a la órbita de Luis Rodríguez San León y por ende, al naciente CDS: «está llevando a cabo una política de desprestigio del actual aparato del Partido [....] y en apoyo, de la política que actualmente está llevando el Presidente de la Diputación y el ex-Senador de UCD [Luis Rodríguez San León]»44; «recorre la Provincia [...] en nombre del ex-senador de nuestro Partido, añadiendo que es quien manda en la Provincia»45; «siempre ha sido un hombre muy adicto a cierto ex-Senador de nuestro partido»46; «es persona que sigue totalmente la línea que le marca el nuevo Partido del Duque»47.

El día 7 de agosto tenía lugar el primer acto del CDS en la provincia, en el que estuvieron presentes Luis Rodríguez San León, quien encabezaba el grupo, César Martín Montes y Jesús Pérez López y, como representante a nivel nacional, Agustín Rodríguez Sahagún ${ }^{48}$. El evento se celebró apenas una semana después de la presentación nacional del CDS en el Hotel Ritz de Madrid. La celeridad en la formación del CDS de Zamora confirmaba la existencia de un grupo perfectamente delimitado, homogéneo y clarificado en la UCD zamorana, cuya división era previa a la escisión suarista. Posteriormente, y tras la adhesión al naciente CDS zamorano de José Miguel López Martínez ${ }^{49}$, se creó la comisión gestora del partido a nivel provincial50, con el fin de presentar oficialmente las candidaturas para las inminentes elecciones que iban a tener lugar en el otoño de $1982^{51}$.

\footnotetext{
43 AHPZ, Fondo UCD, caja 65, Dossier de Expulsados, Acta de la reunión del Comité de Disciplina Provincial del 9 agosto 1982.

44 AHPZ, Fondo UCD, caja 65, Dossier de Expulsados, Informe de la Secretaría Provincial, 5 agosto 1982.

45 AHPZ, Fondo UCD, caja 65, Dossier de Expulsados, Informe de la Secretaría Provincial, 5 agosto 1982.

46 AHPZ, Fondo UCD, caja 65, Dossier de Expulsados, Informe de la Secretaría Provincial, v

47 AHPZ, Fondo UCD, caja 65, Dossier de Expulsados, Informe emitido de la Secretaría de Acción Municipal, 6 agosto 1982.

48 «Rueda de prensa de Agustín Rodríguez Sahagún», in El Correo de Zamora, o8-o8-1982, véase: AGCYL, Gabinete de información, caja 243, Informe Diario $\mathrm{n}^{0} 317$.

49 «Baja en UCD y cese en el Ayuntamiento, en la Diputación y en el Consejo de Castilla y León», in El Correo de Zamora, 5 septiembre 1982, véase: AGCYL, Gabinete de información, caja 242, Informe Diario $\mathrm{n}^{\mathrm{O}} 225$.

50 Además, Luis Rodríguez San León publicó en la prensa local, «Centro Democrático y Social: una vía de esperanza», in El Correo de Zamora, 5 septiembre 1982, véase: AGCYL, Gabinete de información, caja 243, Informe Diario $\mathrm{n}^{0} 317$.

51 «Presentación pública de los candidatos del CDS» in El Correo de Zamora, 23-09-1982; con la asistencia de Fernando Castedo. Véase: AGCYL, Gabinete de información, caja 317, Dossier CDS.
} 


\section{Conclusión}

El presente artículo ha pretendido analizar el papel que las luchas de poder político a nivel provincial tuvieron en la génesis de la descomposición del partido ucedista y el nacimiento del CDS, y lo hemos hecho, resaltando el valor y la utilidad del marco teórico desarrollado por J. Hopkin. En un primer momento, en el caso de Zamora, la lucha por el control político de las estructuras centristas provinciales se entremezcló con un discurso en el que se oponía a los llamados cuneros con los considerados auténticos defensores de los intereses zamoranos. Sin embargo, al hilo del agudizamiento de la crisis nacional de UCD, dichos grupos hubieron de tomar partido por las distintas familias que operaban en el conglomerado centrista. Si al principio, primó la búsqueda de soluciones políticas o "expresiones de voz" (la presentación de dos listas a la III Asamblea de UCD-Zamora, la propuesta de creación de un Comité de Coordinación, etc.), posteriormente, fue la salida del partido la fórmula más viable para solventar el enfrentamiento político.

Podemos concluir, a partir de este estudio de caso, que resulta equívoco afirmar que a nivel provincial fue la diversidad ideológica la principal causa de la descomposición centrista. La mímesis entre el proceso de descomposición nacional y provincial aunque real, lo es más en sus estrategias y ritmos, que en sus motivaciones, pergeñadas de adhesiones personales y clientelares, retóricas localistas, etc. En el caso de la formación del CDS, hemos visto, como la constitución política del suarismo trascendió también la pura discrepancia política e ideológica, solapándose a un proceso de división de las elites políticas locales que lo antecedía temporalmente. Finalmente, a lo largo de este proceso, hemos pretendido resaltar algunos detalles acerca del modus operandi de un partido, que a pesar de gobernar durante más de cinco años, apenas ha dejado testimonios documentales y es que, el precipitado ocaso político de UCD se llevó consigo buena parte de sus vestigios materiales ${ }^{5}$. En definitiva, la singularidad del testimonio documental de los fondos de UCD del Archivo Histórico Provincial de Zamora radica en el hecho de que nos permite reconstruir desde dentro y en profundidad, un proceso, al que en la mayor parte de los casos, tanto a niveles provinciales como nacionales, sólo es posible acceder a través de fuentes indirectas, fundamentalmente, la prensa.

52 «Probable embargo de la sede de UCD [en Murcia] para cumplir una sentencia por despido», in El País, 29 marzo 1983; «Entre tanto, la comisión liquidadora de las siglas centristas, [...], ha puesto en venta, en pública subasta, las sedes locales y provinciales propiedad del partido, con el fin de atender las deudas más inmediatas del mismo», in El País, 23 marzo 1983; «La Telefónica reclama a UCD el pago de una deuda de 11 millones», in El País, 10 abril 1983. 


\section{Fuentes documentales utilizados (Archivos y Hemerotecas)}

AHPZ (Archivo Histórico-Provincial de Zamora), Fondo UCD.

AHPS (Archivo Histórico Provincial de Soria), Fondo UCD.

Archivo General de Castilla y León.

Hemerotecas digitales de El País, ABC y el Periódico de Catalunya.

Archivo Gunther de fuentes orales. 


\section{* El autor}

Darío Diez Miguel está Licenciado en Historia por la Universidad de Valladolid desde 2011 y cursa actualmente el $5^{\circ}$ año de la licenciatura de Filosofía por la UNED. En 2012 realizó el "Máster de Europa y Mundo Atlántico: Poder, Cultura y Sociedad" (Programa de Doctorado del Instituto de Historia Simancas, UVa) y en el año 2013 el "Máster de Formación del Profesorado en la especialidad de $\mathrm{H}^{\mathrm{a}}$, $\mathrm{H}^{\mathrm{a}}$ del Arte y Geografía" (UVa). En la actualidad (desde marzo de 2013) es becario de investigación en la Universidad de Valladolid, donde está realizando el doctorado con un proyecto de tesis titulado: “Adolfo Suárez y el Centro Democrático y Social, 1982-1991".

URL: < http://www.studistorici.com/progett/autori/\#DiezMiguel >

\section{Per citare questo articolo:}

DIEZ MIGUEL, Darío, «Fragmentación política del centrismo Español, 1981-1982. De la UCD al CDS en el caso de Zamora)», Diacronie. Studi di Storia Contemporanea : Periferie. Cultura, economia, politica, 29/3/2014,

URL: < http://www.studistorici.com/2014/3/29/DiezMiguel_numero_17/ >

\section{Diacronie Studi di Storia Contemporanea $\beta$ www.diacronie.it}

Risorsa digitale indipendente a carattere storiografico. Uscita trimestrale. redazione.diacronie@hotmail.it

Comitato di redazione: Jacopo Bassi - Luca Bufarale - Elisa Grandi - Deborah Paci - Fausto Pietrancosta - Matteo Tomasoni - Luca Zuccolo

Diritti: gli articoli di Diacronie. Studi di Storia Contemporanea sono pubblicati sotto licenza Creative Commons 2.5. Possono essere riprodotti a patto di non modificarne i contenuti e di non usarli per fini commerciali. La citazione di estratti è comunque sempre autorizzata, nei limiti previsti dalla legge. 